ORIGINAL PAPER

\title{
BRCA1 AND PARP1 MRNA EXPRESSION DURING \\ PROGRESSION FROM NORMAL BREAST TO DUCTAL CARCINOMA IN \\ SITU AND INVASIVE BREAST CANCER: A LASER MICRODISSECTION \\ STUDY
}

Jolanta Hybiak, Pawel Domagala, Wenancjusz Domagala

Department of Pathology, Pomeranian Medical University, Szczecin, Poland

\begin{abstract}
The contribution of DNA damage repair mechanisms to the progression of normal breast to ductal carcinoma in situ (DCIS) and invasive ductal carcinoma is largely unknown. The purpose of this report was to assess the mRNA expression levels of two important genes associated with DNA repair, BRCA1 and PARP1, in normal breast tissue, DCIS G1, G2 and G3, and co-existing adjacent invasive ductal carcinoma. BRCA1 and PARP1 mRNA expression was assessed in 32 ductal carcinomas in situ of the breast using a laser microdissection and pressure catapulting system and quantitative real-time PCR. The relative expression of BRCA1 mRNA was significantly increased in DCIS G2 and DCIS G3 relative to normal breast tissue ( $\mathrm{p}=0.02, \mathrm{p}=0.001$, respectively). Significant differences in BRCA1 expression were observed between DCIS G1 and G2 $(\mathrm{p}=0.02)$ and between DCIS $\mathrm{G} 1$ and $\mathrm{G} 3(\mathrm{p}=0.0007)$. No significant differences in BRCA1 expression were observed between normal breast tissue and DCIS G1 and between DCIS component and adjacent invasive ductal carcinoma. No significant differences in the relative expression of PARP1 mRNA were observed between groups. Increased BRCA1 mRNA expression (but not PARP1 mRNA) occurs early in the development of breast cancer, i.e. at the noninvasive (DCIS) stage, suggesting a demand for increased activity of a DNA double-strand break repair by homologous recombination. DCIS G1 and normal breast tissue share highly similar BRCA1 and PARP1 expression level. This finding supports the idea that DCIS G1 belongs to a separate family of precursor lesions with low malignant potential.
\end{abstract}

Key words: breast cancer, BRCA1, PARP1, laser microdissection, DCIS.

\section{Introduction}

In recent years, due to mammography and increased public awareness, ductal carcinoma in situ (DCIS) has been diagnosed with increased frequency. In 2017, approximately 63,410 new cases of DCIS were diagnosed in the U.S. [1]. DCIS is a noninvasive (preinvasive) lesion, a type of intraepithelial neoplasia, characterised by the growth of cancer cells that have not invaded the basement membrane of a duct and the nearby connective tissue but remain and proliferate within the epithelium of a breast duct. DCIS is generally accepted to be the direct precursor of invasive ductal carcinoma (IDC); however, DCIS has "an inherent but not necessarily obligate tendency for progression to invasive breast carcinoma" [2]. Approximately $50 \%$ of DCIS lesions are estimated to progress to invasive breast cancer if left untreated [3]. 
Histologically DCIS is divided into three grades: low (G1), intermediate (G2), and high (G3), based on nuclear grade alone or in conjunction with necrosis and/or cell polarisation [2]. Recent evidence has suggested that low-grade DCIS and high-grade DCIS are distinct lesions, although progression from low- to high-grade lesions may occur [4]. Low-grade DCIS and high-grade DCIS are believed to arise from two distinct molecular pathways (for review see [5]). Furthermore, low-grade DCIS generally progresses to low-grade IDC, whereas high-grade DCIS progresses to high-grade IDC. The transition from the noninvasive (preinvasive) stage to invasive cancer is believed to be associated with quantitative rather than qualitative differences in gene expression [6]. Several breast cancer gene expression studies have established that the expression profile of low-grade DCIS is populated by genes that are associated with oestrogen receptor expression, whereas the expression profile of high-grade DCIS includes genes associated with cell cycle and mitotic activity [5]. A better understanding of DCIS at the molecular level may help to devise strategies to arrest the disease at this noninvasive stage of progression. In recent years, an effort has been made to classify DCIS not by its ability to recur after surgical treatment (as has been standard practice) but by its ability to progress to IDC [7].

Genes that encode proteins involved in DNA repair are important targets in the multistep process of carcinogenesis. Unrepaired DNA damage can result in unregulated cell growth and cancer [8]. However, although various aspects of progression from DCIS to IDC have been extensively studied (for review see [9]) BRCA1 and PARP1 (Poly[ADP-ribose] polymerase 1) expression status during this transition is largely unknown. In a recent thorough review discussing the role of genetic alterations and changes in microenvironment in progression from DCIS to IDC, the significance of expression of genes engaged in DNA damage repair is not even mentioned [9]. To date, there is only one report in which changes in BRCA1 and PARP1 expression along normal breast/DCIS/IDC progression axis were detected [10].

The purpose of this report was to assess the mRNA expression levels of two important genes associated with DNA repair, BRCA1 and PARP1, in normal breast tissue, DCIS G1, G2 and G3, and co-existing adjacent IDC. To date, BRCA1 $\mathrm{mRNA}$ and PARP1 mRNA expression levels in various grades of DCIS have not been reported (nor compared with co-existing adjacent IDC).

\section{Material and methods}

\section{Patients}

The study was based on tumour tissue from 32 unselected patients with DCIS. The following tissues were studied: a) 19 cases of DCIS with a co-existing adjacent invasive ductal carcinoma (DCIS-in-IDC), including four DCIS G1, eight DCIS G2, and seven DCIS G3; b) 13 ductal carcinomas in situ only (pure DCIS), including two DCIS G1, three DCIS G2, and eight DCIS G3; and c) five normal breast tissue samples (microdissected terminal duct lobular units). All breast cancer specimens were obtained between 2011 and 2013 in Szczecin, Poland. The mean age of the patients was 59 years, and none of the patients had received endocrine therapy, chemotherapy, or radiotherapy prior to surgery. Patients with IDC were tested for three founder germline mutations in BRCA1 (C61G, 4153delA, and 5382insC) described in the Polish population [11] and none possessed germline mutations. The study was approved by the Ethics Committee of the Pomeranian Medical University in Szczecin, Poland.

\section{Tumour pathology}

DCIS grading was conducted by consensus of two experienced breast pathologists (WD, PD), according to the nuclear grade criteria listed in the WHO 2012 grading scheme [2]. Pure DCIS samples were tumour cells captured from ducts of pure DCIS lesion; DCISin-IDC samples were tumour cells captured from the in situ (DCIS) component of the co-existing adjacent IDC; and IDC samples were tumour cells captured from the invasive cancer adjacent to the in situ component. Invasive breast cancer grade was classified according to the Elston \& Ellis grading system [12].

\section{Laser microdissection}

Three serial $5-\mu \mathrm{m}$-thick sections were cut from each tissue specimen fixed in $10 \%$ neutral buffered formalin and embedded in paraffin (FFPE). The sections were mounted onto glass slides covered with PEN membrane (Zeiss, Bernried, Germany) and stained with $0.1 \%(\mathrm{w} / \mathrm{v})$ Cresyl Violet acetate (Sigma, Saint Louis, USA) in $50 \%$ alcohol. All steps of the procedure were conducted under RNase-free conditions with care to avoid contamination. The PALM MicroBeam (Zeiss) laser microdissection and pressure catapulting (LMPC) system was used according to the manufacturer's instructions. Areas of interest (DCIS, IDC, or benign epithelium) were excised with a laser and catapulted into an adhesive cap. Selected elements were supervised under a microscope to ensure tissue homogeneity. Representative images of DCIS before and after microdissection are presented in Fig. 1A-F. 

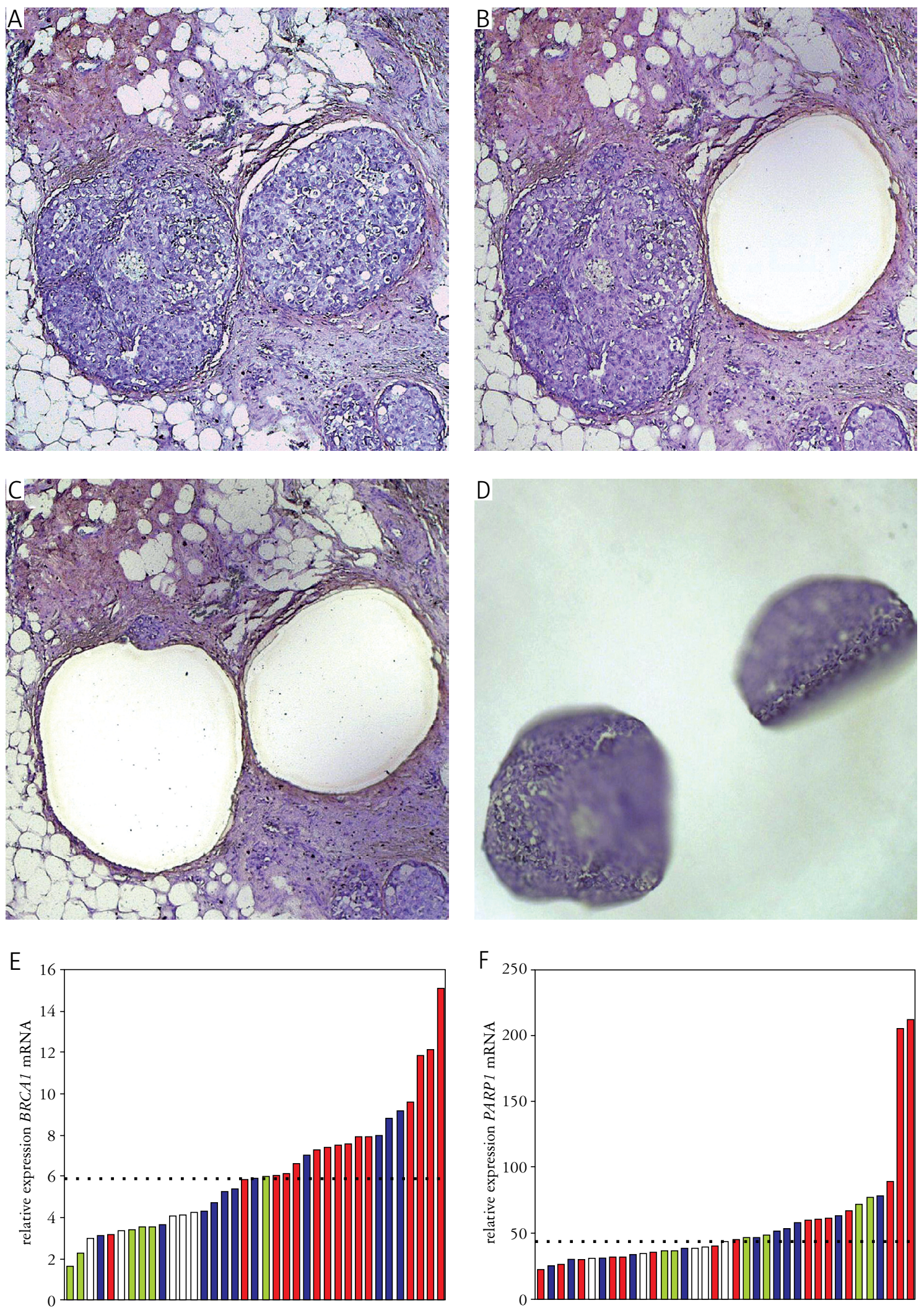

Fig. 1A-F. Example of laser microdissection of DCIS lesions and relative expression of BRCA1 mRNA and PARP1 mRNA. A) Laser microdissection of two ducts with DCIS before microdissection. B) Empty space at the site of DCIS that had been removed. C) Empty spaces after the microdissection of both DCIS lesions. D) Tissue of both microdissected DCIS lesions captured in an adhesive cap. Relative expression of E) BRCA1 mRNA and F) PARP1 mRNA in 32 DCIS samples and 5 normal breast samples. DCIS G1 - green bar; DCIS G2 - blue bar; DCIS G3 - red bar; normal breast tissue - white bar. The dotted line represents the median 


\section{RNA isolation and cDNA synthesis}

Total RNA was extracted using the RNeasy FFPE Kit (Qiagen, Hilden, Germany) according to the manufacturer's instructions with LMPC-specific modifications introduced by Zeiss Microscopy Lab. Microdissected tissue was digested overnight at $56^{\circ} \mathrm{C}$ with $10 \mu \mathrm{l}$ of proteinase $\mathrm{K}$ and $150 \mu \mathrm{l}$ of PKD buffer. To reverse formaldehyde modifications samples were incubated at $80^{\circ} \mathrm{C}$ for $15 \mathrm{~min}$. DNA was removed by incubation of the lysate with $10 \mu \mathrm{l}$ of DNase I and $16 \mu \mathrm{l}$ of DNase Booster Buffer at room temperature for $15 \mathrm{~min}$. In the next step, the lysate was transferred to a new $1.5-\mathrm{ml}$ microcentrifuge tube, and $320 \mu \mathrm{l}$ of RBC buffer and $720 \mu \mathrm{l}$ of ethanol $99.6 \%$ were added. $700 \mu \mathrm{l}$ of the sample was transferred to a RNeasy MinElute spin column placed in a $2-\mathrm{ml}$ collection tube and centrifuged for $15 \mathrm{sec}$ at 10,000 rpm; flow-through was discarded. This step was repeated. To wash the spin column membrane, $500 \mu \mathrm{l}$ of RPE buffer was added and centrifuged for $15 \mathrm{sec}$ at 10,000 rpm; flow-through was discarded. Next, $500 \mu \mathrm{l}$ of RPE buffer was added again and centrifuged for $2 \mathrm{~min}$ at $10,000 \mathrm{rpm}$. To dry the spin column membrane, the column was placed in new $2-\mathrm{ml}$ collection tubes and centrifuged for $5 \mathrm{~min}$ at full speed. For elution, $14 \mu \mathrm{l}$ of RNase-free water was used.

Sample quantity was assessed with an Agilent 2100 Bioanalyzer RNA 6000 Pico Assay (Agilent, St Clara, USA). RNA (20 ng) from the microdissected tissue was converted to cDNA using a Transcriptor First Strand cDNA Synthesis Kit (Roche Applied Science, Mannheim, Germany) and random hexamer priming. RNA without reverse transcriptase was used as a negative control (RT-).

\section{Selection of primers and probes}

To interpret the quantitative gene expression measurements, HPRT1 was used as a reference gene [13]. A limited amount of RNA after DCIS laser microdissection from FFPE does not allow for normalisation with multiple genes. De Kok et al. [14] identified HPRT1 as the single reference gene that could replace the measurement of multiple genes in breast tissue, ranging from normal tissues to metastatic carcinomas. HPRT1 was also frequently present in the best combination of reference genes [13]. Primers and probe sequences for BRCA1, PARP1, and HPRT1 available in the literature $[15,16,17,18$, $19,20]$ were tested in three stages, taking into account the requirements of FFPE material analysis.

$1^{\text {st }}$ step: In silico validation. Basic primer parameters were checked with Primer3 software according to the checklist for real-time PCR assay validation [21]. The BLAST algorithm [22] and Human Genomic Plus Transcript database were used to confirm primers' and probes' specificity to target genes. To avoid gDNA amplification, primers were targeted to different exons or span introns. Special attention was paid to keep amplicons no longer than $100 \mathrm{bp}$. Expected PCR efficiency was assessed with PcrEfficiency software [23].

$2^{\text {nd }}$ step: Real-time PCR with SYBR Green. The absence of amplification artefacts (primers dimers, hairpin, and gDNA amplification) was confirmed by real-time PCR with SYBR Green I (Roche) with a melting curve analysis. Reactions were performed on material extracted from FFPE samples prepared in the same manner as the analysed samples.

$3^{\text {rd }}$ step: Real-time PCR with hydrolysis probes. Based on serial dilutions, the reaction efficiency of each assay was determined by real-time PCR with probes. The reaction was run for 50 cycles, to be certain that NTC- and RT-controls were negative.

For BRCA1 and HPRT1 genes, sequences of primers and probes that met the above requirements were selected from the literature $[17,18]$, whereas for $P A R P 1$, none of the sequences available in the literature fulfilled these requirements. To choose the best primer set for PARP1, software of the UPL Probe Assay Design Centre (Roche) was used. The primer and probe sequences are presented in Table I.

Table I. Primers and probes used in the present study

\begin{tabular}{|c|c|c|c|}
\hline GENE & REFSEQ & SEQUENCES 5' TO 3' & AMPLICON \\
\hline \multirow[t]{3}{*}{ HPRT1 } & \multirow[t]{3}{*}{ NM_000194.2 } & F:5'-GGCAGTATAATCCAAAGATGGTCAA-3' & \multirow[t]{3}{*}{$80 \mathrm{bp}$} \\
\hline & & R:5'-GTCTGGCTTATATCCAACACTTCGT-3' & \\
\hline & & P:5'-FAM-CAAGCTTGCTGGTGAAAAGGACCCC-BHQ1-3' & \\
\hline \multirow[t]{3}{*}{$B R C A 1$} & \multirow[t]{3}{*}{ NM_007294.4 } & F:5'-CAGAGGACAATGGCTTCCATG-3' & \multirow[t]{3}{*}{$81 b p$} \\
\hline & & R:5'-CTACACTGTCCAACACCCACTCTC-3' & \\
\hline & & P:5'-FAM-AATTGGGCAGATGTGTGAGGCACCTG-BHQ1-3' & \\
\hline \multirow[t]{3}{*}{ PARP1 } & \multirow[t]{3}{*}{ NM_001618.3 } & F:5'-CTGGAGGACGACAAGGAAAA-3' & \multirow[t]{3}{*}{$76 \mathrm{bp}$} \\
\hline & & R:5'-TGTTGCTACCGATCACCGTA-3’ & \\
\hline & & P:5'-FAM-CTGGGGCC-Q-3' & \\
\hline
\end{tabular}




\section{Quantitative real-time PCR reaction}

Quantitative real-time PCR (qPCR) was performed on a Light-Cycler 480 II instrument (Roche) with a hydrolysis probe as the detection format. Two microlitres of cDNA were added to a $20-\mu \mathrm{l}$ reaction containing $0.5 \mu \mathrm{M}$ of each primer and $0.2 \mu \mathrm{M}$ of probe and LightCycler 480 Probes Master (Roche). The temperature protocol was $10 \mathrm{~min}$ at $95^{\circ} \mathrm{C}$ followed by 50 cycles of amplification at $95^{\circ} \mathrm{C}$ for $10 \mathrm{~s}, 60^{\circ} \mathrm{C}$ for $10 \mathrm{~s}$, and $72^{\circ} \mathrm{C}$ for $1 \mathrm{~s}$. The reaction for each gene was performed in triplicate with the RT- and NTC-negative controls. Cycle of quantification $(\mathrm{Cq})$ values were determined by the second derivative maximum method. Relative quantification was calculated with the E-method based on the efficiency correction for each assay.

\section{Statistical analysis}

Differences in the distributions of mRNA expression between the two patient groups were analysed with the Mann-Whitney U test. The $\chi^{2}$ test was used when expression was categorised as "low" or "high" based on the median of relative expression. A Wilcoxon-Pratt test was used to analyse different expression levels between paired IDC and in situ components from the same tumour. Statistical analyses were performed using GraphPad Prism v 6.03 (GraphPad Software, La Jolla, CA, USA). All p values were twotailed, and $95 \%$ confidence intervals were adopted. $\mathrm{P}<0.05$ was considered significant.

\section{Results}

\section{BRCA1 mRNA in DCIS and IDC}

The distribution of $B R C A 1 \mathrm{mRNA}$ expression levels among DCIS G1, G2, G3, and normal breast tissue samples is presented in Fig. 1E. There were statistically significant differences in the relative expression of BRCA1 mRNA between the groups studied. Compared with normal breast tissue, the relative expression of BRCA1 mRNA was significantly increased in DCIS ( $\mathrm{p}=0.02)$ and IDC $(\mathrm{p}=0.02)$ (Fig. 2A). However, when DCIS was divided by grade, the relative expression of $B R C A 1 \mathrm{mRNA}$ was only increased in DCIS G2 (1.6-fold) and DCIS G3 (2.2-fold) compared with that in normal breast tissue $(p=0.02$ and $\mathrm{p}=0.001$, respectively; Fig. 2B). Conversely, no significant difference in BRCA1 expression was found between normal breast tissue and DCIS G1 $(\mathrm{p}=0.53)$. Significant differences in BRCA1 expression were observed between DCIS G1 and G2 (1.7-fold increase, $p=0.02$ ) and DCIS G1 and G3 (2.3-fold increase, $\mathrm{p}=0.0007)$. The difference between DCIS G2 and G3 was on the verge of statistical significance (1.4-fold increase, $\mathrm{p}=0.047$; Fig. 2B).
When BRCA1 mRNA expression levels in the group of all DCIS samples $(\mathrm{n}=32)$ was compared with the IDC group ( $n=19)$, no significant differences were observed ( $\mathrm{p}=0.43$ ) (Fig. 2A). Furthermore, when the pure DCIS $(\mathrm{n}=13)$ and DCIS-in-IDC groups $(\mathrm{n}=19)$ were compared separately with IDC ( $\mathrm{n}=19)$, no significant differences were seen between the IDC and DCIS-in-IDC ( $p=0.09$ ), IDC and pure DCIS groups $(\mathrm{p}=0.61)$, or pure DCIS and DCIS-in-IDC groups $(\mathrm{p}=0.81)$. Although the expression of $B R C A 1 \mathrm{mRNA}$ in IDC was 1.3 -fold higher than in the adjacent DCIS (DCIS-in-IDC), the difference was not significant $(\mathrm{p}=0.09)$.

There was a linear dependence between DCIS grade and the level of $B R C A 1$ mRNA ( $\mathrm{p}=0.0005$, Table II). High BRCA1 expression levels (above median) were significantly associated with DCIS G3 lesions. High BRCA1 expression was found in $80 \%$ $(12 / 15)$ of G3 DCIS lesions but only in $36.4 \%$ (4/11) of G2 DCIS lesions and no G1 DCIS lesions $(\mathrm{p}=0.002$, Table II).

\section{PARP1 mRNA in DCIS and IDC}

The distribution of PARP1 mRNA expression among DCIS G1, G2, G3, and normal breast tissue samples is shown in Fig. 1F. No significant differences were observed in the relative expression of $P A R P 1$ mRNA between any groups. No differences were observed between normal breast tissue and DCIS or IDC (Fig. 2C) or between normal breast tissue and various DCIS grades (normal breast vs. DCIS G1 [p $=0.13]$, vs. G2 [p =0.49] and vs. G3 [p = 0.37]) or between DCIS grades (DCIS G1 vs. G2 [p $=0.54]$, DCIS G1 vs. G3 [p = 0.63]; DCIS G2 vs. G3 [p = 0.62]) (Fig. 2D).

No significant differences in PARP1 mRNA expression were observed between matched IDC lesions and adjacent DCIS lesions (DCIS-in-IDC) $(\mathrm{p}=0.79)$, IDC lesions and pure DCIS lesions $(p=0.80)$, or pure DCIS and DCIS-in-IDC ( $p=0.72)$.

Table II. Level of BRCA1 and PARP1 mRNA in DCIS according to grade

\begin{tabular}{lccc}
\hline BRCA1 MRNA & Low $^{A} \mathbf{N}(\%)$ & HIGH $^{\mathrm{N}}(\%)$ & $\mathbf{P}$ \\
\hline DCIS G1 & $6(100)$ & $0(0)$ & \\
\hline DCIS G2 & $7(63.6)$ & $4(36.4)$ & \\
\hline DCIS G3 & $3(20)$ & $12(80)$ & 0.002 \\
\hline $\boldsymbol{P A R P 1}$ MRNA & & & \\
\hline DCIS G1 & $3(50)$ & $3(50)$ & \\
\hline DCIS G2 & $5(45.5)$ & $6(54.5)$ & \\
\hline DCIS G3 & $8(53.3)$ & $7(46.7)$ & 0.92 \\
\hline${ }^{a} \leq$ median & & & \\
${ }^{b}>$ median & & &
\end{tabular}


A

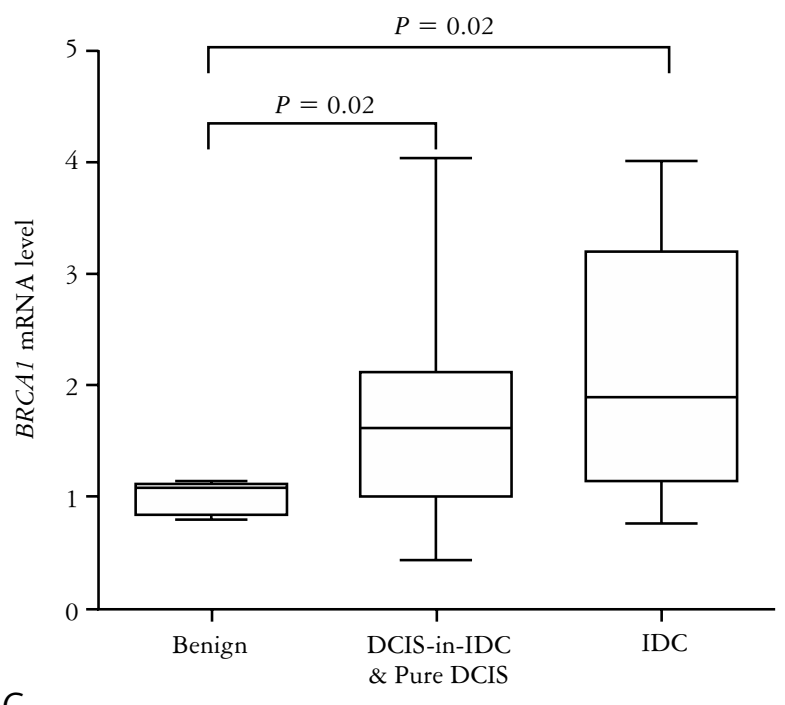

C

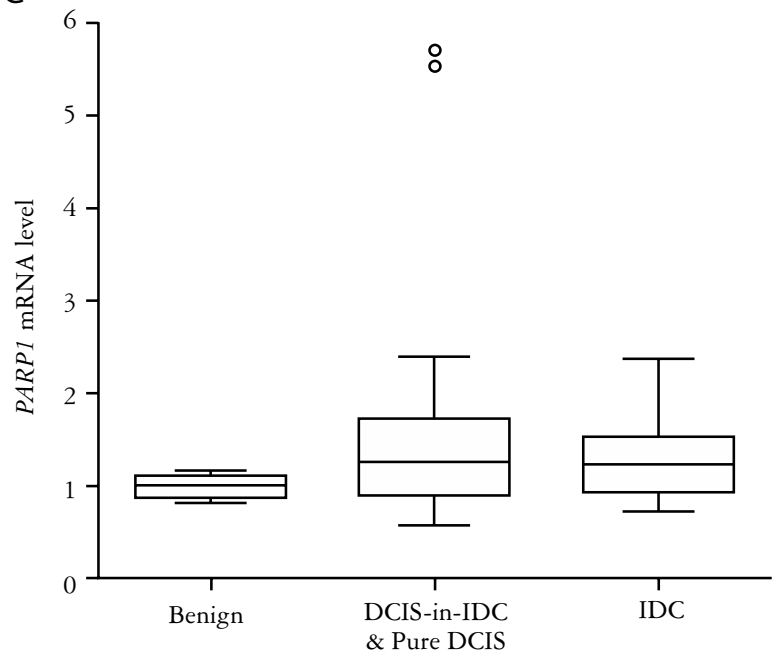

B

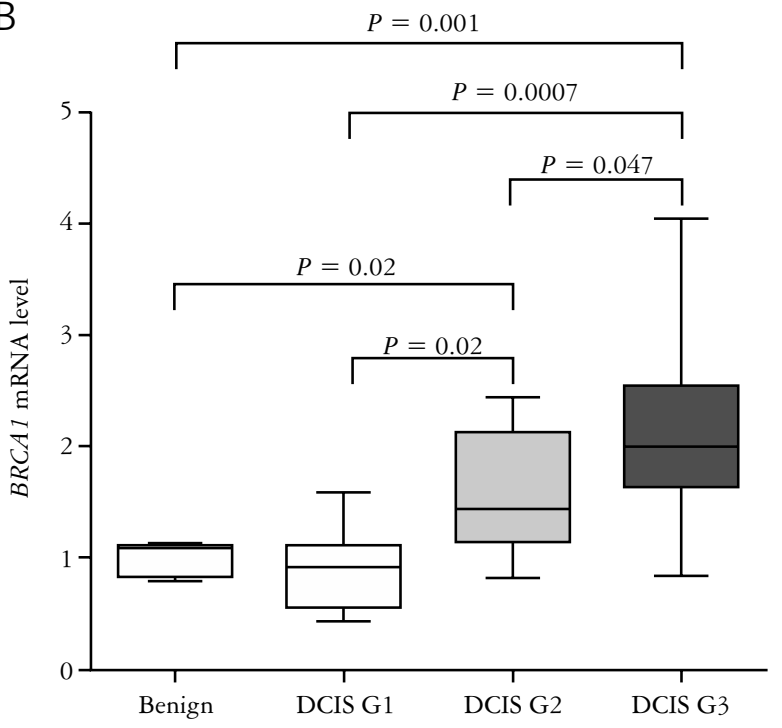

D

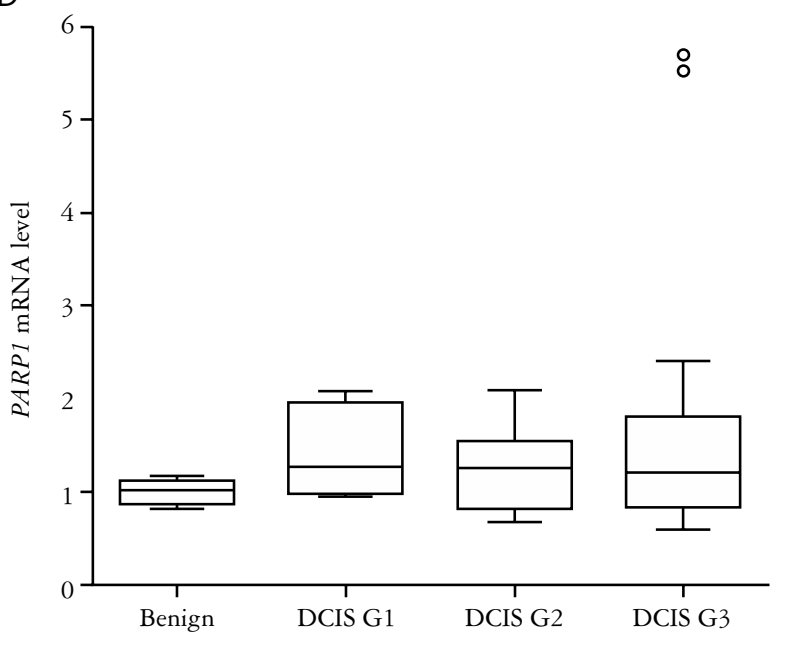

Fig. 2A-D. Relative BRCA1 and PARP1 mRNA expression in normal breast tissue (benign), DCIS (DCIS-in-IDC and pure DCIS) and IDC. A) Relative BRCA1 mRNA expression in normal breast tissue (benign), DCIS (DCIS-in-IDC and pure DCIS) and IDC. B) Relative BRCA1 mRNA expression in normal breast tissue (benign) and DCIS G1, G2, and G3. C) Relative PARP1 mRNA expression in normal breast tissue (benign), DCIS (DCIS-in-IDC and pure DCIS) and IDC. D) Relative PARP1 mRNA expression in normal breast tissue (benign) and DCIS G1, G2, and G3. The boxes contain $50 \%$ of the values. The upper and lower borders indicate the $25^{\text {th }}$ and $75^{\text {th }}$ percentiles, respectively. The upper and lower whiskers indicate the highest and lowest value, respectively. The dots indicate outliers (Tukey plot). The mean BRCA1 or PARP1 mRNA expression level in benign tissue was set at 1

No significant associations were found between PARP1 expression levels and DCIS grade. A high level of $P A R P 1$ expression (above median) was found in $46.7 \%(7 / 15)$ of DCIS G3 lesions, $54.5 \%(6 / 11)$ of DCIS G2, and $50 \%(3 / 6)$ of DCIS G1 ( $\mathrm{p}=0.92$, Table II).

\section{Association between PARP1 and BRCA1 mRNA expression along normal breast/DCIS/IDC progression axis}

The ratio of PARP1 to BRCA1 mRNA expression decreased during progression from benign tissue (9.9-fold) to DCIS (8.8-fold) to IDC (6.2-fold), largely because the expression of $B R C A 1 \mathrm{mRNA}$ increased whereas the PARP1 mRNA expression remained relatively stable.

\section{Discussion}

In order to survive, DCIS cells have to adapt to the unfavourable intraductal environment, which is hypoxic and far from blood vessels that are separated from the duct interior by basement membrane. Hence the cells are under persistent severe metabolic and hypoxic stress, which may promote genetic instability and the selection of neoplastic cells with invasive 
potential (for review see [24]). Failure to repair DNA damage induced by impaired cellular proliferation, hypoxia, or carcinogens may lead to mutations in proto-oncogenes and tumour suppressor genes and genomic instability. Therefore, efficient DNA repair mechanisms are essential to protect cells against mutations in cancer-related genes [25]. Surprisingly, no study has assessed BRCA1 and PARP1 mRNA levels in DCIS and adjacent IDC or in various histological grades of DCIS to date, although both genes are known to be involved in DNA damage repair and to play an important role in malignant transformation. $B R C A 1$ plays a critical role in the repair of DNA double-strand breaks by a high-fidelity mechanism of homologous recombination [26]. Loss of BRCA1 function may result in the accumulation of ER-negative stem/progenitor cells [27]. PARP1 is an enzyme engaged in the repair of DNA single-strand breaks and double-strand breaks [28]. The vast majority of invasive breast carcinomas expressed high levels of nuclear PARP1 protein [29].

\section{BRCA1 mRNA expression in DCIS and co-existing adjacent IDC}

We found increased expression of BRCA1 mRNA, but not PARP1 mRNA, during progression along the axis from normal breast tissue through increasing histological grades of DCIS. Furthermore, analysis of laser microdissected samples of DCIS lesions revealed significant correlation between the increased expression of BRCA1 mRNA and DCIS histological grade. High $B R C A 1$ expression levels were significantly associated with G3 DCIS lesions (80\% of DCIS G3 vs. no DCIS G1). Similarly, a positive correlation between genomic instability and histological grade of DCIS has been reported in the literature. The overall frequency of allelic imbalance increased significantly with increasing grade and was significantly higher in poorly differentiated lesions compared with well-differentiated or moderately differentiated lesions [30]. The level of genomic instability as assessed by dysregulation in telomere content and the extent of allelic imbalance also increased with progression from normal breast tissue to DCIS [31]. A tendency for association of short telomeres with high-grade DCIS lesions has been found [32]. DNA methylation levels increased significantly during the progressive stages of breast cancer development [33].

Thus, the increased expression of $B R C A 1 \mathrm{mRNA}$ (but not PARP1 mRNA) observed in our study with progression from normal breast tissue to DCIS G3 parallels the increasing levels of genomic instability reported in the literature along this axis and appears to suggest a demand for increased activity of a DNA double-strand break repair by a high-fidelity mechanism of homologous recombination. The increased BRCA1 mRNA expression that occurs at the pre-invasive (DCIS) stage may play a role in carcinogenesis. It is tempting to speculate that the efficiency of BRCA1-associated DNA damage repair system may modify the risk and speed of the transition from DCIS to invasive cancer. In fact, mutations in $B R C A 1$ have been associated with genomic instability and malignant transformation [34]. Although most DCIS lesions will not progress to IDC, some do; thus, there is a need to identify risk factors. Whether the BRCA1 mRNA expression level in DCIS may reflect a risk of progression to invasive disease requires further research.

We observed similar BRCA1 mRNA expression levels in DCIS and co-existing adjacent IDC. Lack of significant differences in BRCA1 mRNA expression between DCIS and IDC is consistent with reports documenting that the level of genomic instability did not differ significantly between DCIS and invasive carcinomas [31]. Very few studies have analysed DCIS/IDC transitions at the molecular level comparing DCIS with matched adjacent IDC. The results of these studies led to the general conclusion that there is remarkable genomic and transcriptomic similarity between DCIS and IDC. Schuetz et al. [35] analysed nine patients and identified a set of progression-specific candidate genes using laser microdissection and microarray analysis. Hernandez et al. [36] used a set of 13 DCIS lesions and found similar genomic profiles of matched DCIS and invasive cancers, although somatic mutations and copy number aberrations varied in three matched pairs. When analysed as separate groups, DCIS and adjacent IDC lesions did not exhibit any significant differences in terms of copy number aberrations and mutations in known cancer genes [36]. Van Hoesel et al. [33] analysed 23 DCIS lesions and synchronous invasive cancers and found no significant difference in the methylation index between DCIS and invasive cancer. Heselmeyer-Haddad et al. [37] examined single cells microdissected from matched DCIS and invasive cancer samples and reported that matched DCIS and IDC had similar but not identical patterns of genomic imbalances. Meeker et al. [32], based on 14 cases of matched DCIS and IDC, reported that most telomere shortening in cases of concurrent DCIS and IDC occurred at the DCIS stage because telomeres were similarly shortened in both components. Castro et al. [7] studied 11 cases of co-existing DCIS and IDC and found very similar molecular profiles of cells from DCIS-in-IDC and cells from IDC. Balleine et al. [38] examined a group of 45 DCIS samples with concomitant invasive cancer and presented gene expression-based classification of DCIS into low- and high-grade lesions. BRCA1 mRNA expression in our study also largely divided DCIS into low(G1) and high-grade (G2+3) lesions. Our results are in partial disagreement with the report of Volinia 
et al. [10], who analysed 17 DCIS lesions and 118 IDC lesions and reported a small set of genes, including $B R C A 1$ and PARP1, that were activated in DCIS and down-regulated in invasive cancer. However, the DCIS samples they studied were not matched with adjacent IDC lesions, and the grade of DCIS lesions was not provided.

\section{BRCA1 mRNA expression in normal breast vs. DCIS G1}

Contrary to the difference between the normal breast tissue and DCIS G2+G3 groups, no significant difference in BRCA1 mRNA expression was observed between normal breast tissue and DCIS G1, suggesting a low level of genetic instability in DCIS G1 that does not require increased involvement of a highfidelity mechanism of DNA damage repair. This finding supports the idea that DCIS G1 belongs to a separate family of precursors with low malignant potential $[39,40]$. There has been concern that some low-risk DCIS lesions may be overtreated, and some low-risk DCIS lesions have been suggested to require only "watchful waiting" or to be treated by endocrine therapy alone if ER-positive (for review, see [41]). However, given the evidence that after surgery alone, invasive recurrence rates are approximately $10-20 \%$ at 15 years [42], it is difficult to define such low-risk DCIS lesions. Whether low BRCA1 mRNA expression, similar to normal breast tissue, could be one of the defining characteristics of low-risk DCIS requires further research.

We used LMPC to obtain a pure population of tumour cells from DCIS and IDC samples because LMPC is the most accurate technique to separate tumour cells from benign stromal cells. Various (genetic and epigenetic) characteristics of progression from normal breast to invasive cancer have been studied with laser microdissection $[7,33,38,43]$. These studies provided important information despite the fact that only small groups of tumours containing DCIS and adjacent IDC (9-45 cases) have been analysed. Our study supports the applicability of laser microdissection for studying the mRNA expression of various genes in pure populations of cells from DCIS in FFPE samples.

In conclusion, increased $B R C A 1$ mRNA expression (but not PARP1 mRNA) occurs early in the development of breast cancer, i.e. at the noninvasive (DCIS) stage, suggesting a demand for increased activity of DNA double-strand break repair by homologous recombination. Lack of significant differences in BRCA1 or PARP1 expression between normal breast tissue and DCIS G1 supports the idea that DCIS G1 belongs to a separate family of lesions with low malignant potential.

The authors declare no conflict of interest.

\section{References}

1. Siegel RL, Miller KD, Jemal A. Cancer statistics, 2017. CA Cancer J Clin 2017; 67: 7-30.

2. Schnitt SJ, Allred C, Britton P, et al. Ductal carcinoma in situ. In: WHO Classification of Tumours of the Breast, Lakhani SR, Ellis IO, Schnitt SJ, Tan PH, van de Vijver MJ (eds). IARC, Lyon 2012; 90-94

3. Thompson A, Brennan K, Cox A, et al. Evaluation of the current knowledge limitations in breast cancer research: a gap analysis. Breast Cancer Res 2008; 10: R26.

4. Allred DC, Wu Y, Mao S, et al. Ductal carcinoma in situ and the emergence of diversity during breast cancer evolution. Clin Cancer Res 2008; 14: 370-378.

5. Bombonati A, Sgroi DC The molecular pathology of breast cancer progression. J Pathol 2011; 223: 307-317.

6. Ma XJ, Salunga R, Tuggle JT, et al. Gene expression profiles of human breast cancer progression. Proc Natl Acad Sci U S A 2003; 100: 5974-5979.

7. Castro NP, Osorio CA, Torres C, et al. Evidence that molecular changes in cells occur before morphological alterations during the progression of breast ductal carcinoma. Breast Cancer Res 2008; 10: R87.

8. Goode EL, Ulrich CM, Potter JD. Polymorphisms in DNA repair genes and associations with cancer risk. Cancer Epidemiol Biomarkers Prev 2002; 11: 1513-1530.

9. Cowell CF, Weigelt B, Sakr RA, et al. Progression from ductal carcinoma in situ to invasive breast cancer: revisited. Mol Oncol 2013; 7: 859-869.

10. Volinia S, Galasso M, Sana ME, et al. Breast cancer signatures for invasiveness and prognosis defined by deep sequencing of microRNA. Proc Natl Acad Sci U S A 2012; 109: 30243029.

11. Lubinski J, Korzen M, Gorski B, et al. Breast cancer susceptibility genes. J BUON 2007; 12 Suppl 1: S23-S29.

12. Elston CW, Ellis IO. Pathological prognostic factors in breast cancer. I. The value of histological grade in breast cancer: experience from a large study with long-term follow-up. Histopathology 1991; 19: 403-410.

13. Vandesompele J, De Preter K, Pattyn F, et al. Accurate normalization of real-time quantitative RT-PCR data by geometric averaging of multiple internal control genes. Genome Biol 2002; 3: research0034.1-research0034.

14. de Kok JB, Roelofs RW, Giesendorf BA, et al. Normalization of gene expression measurements in tumor tissues: comparison of 13 endogenous control genes. Lab Invest 2005; 85: 154-159.

15. To MD, Done SJ, Redston M, et al. Analysis of mRNA from microdissected frozen tissue sections without RNA isolation. Am J Pathol 1998; 153: 47-51.

16. Egawa C, Miyoshi Y, Taguchi T. Quantitative analysis of BRCA1 and BRCA2 mRNA expression in sporadic breast carcinomas and its relationship with clinicopathological characteristics. Jpn J Cancer Res 2001; 92: 624-630.

17. Specht K, Richter T, Muller U, et al. Quantitative gene expression analysis in microdissected archival formalin-fixed and paraffin-embedded tumor tissue. Am J Pathol 2001; 158: 419-429.

18. Chalabi N, Le Corre L, Maurizis JC, et al. The effects of lycopene on the proliferation of human breast cells and BRCA1 and BRCA2 gene expression. Eur J Cancer 2004; 40:1768-1775.

19. Clark-Langone KM, Wu JY, Sangli C, et al. Biomarker discovery for colon cancer using a 761 gene RT-PCR assay. BMC Genomics 2007; 8: 279.

20. Margeli M, Cirauqui B, Castella E, et al. The prognostic value of BRCA1 mRNA expression levels following neoadjuvant chemotherapy in breast cancer. PLoS One 2010; 5: e9499.

21. Raymaekers M, Smets R, Maes B, et al. Checklist for optimization and validation of real-time PCR assays. J Clin Lab Anal 2009; 23: 145-151. 
22. NCBI Resource Coordinators. Database resources of the National Center for Biotechnology Information. Nucleic Acids Res 2013; 41: D8-D20.

23. Mallona I, Weiss J, Egea-Cortines M. pcrEfficiency: a Web tool for PCR amplification efficiency prediction. BMC Bioinformatics $2011 ; 12: 404$

24. Espina V, Mariani BD, Gallagher RI, et al. Malignant precursor cells pre-exist in human breast DCIS and require autophagy for survival. PLoS One 2010; 5: e10240.

25. Hoeijmakers JH Genome maintenance mechanisms for preventing cancer. Nature 2001; 411: 366-374.

26. Narod SA, Foulkes WD. BRCA1 and BRCA2: 1994 and beyond. Nat Rev Cancer 2004; 4: 665-676.

27. Liu S, Ginestier C, Charafe-Jauffret E, et al. BRCA1 regulates human mammary stem/progenitor cell fate. Proc Natl Acad Sci U S A 2008; 105: 1680-1685.

28. De Vos M, Schreiber V, Dantzer F. The diverse roles and clinical relevance of PARPs in DNA damage repair: current state of the art. Biochem Pharmacol 2012; 84: 137-146.

29. Domagala P, Huzarski T, Lubinski J, et al. PARP-1 expression in breast cancer including BRCA1-associated, triple negative and basal-like tumors: possible implications for PARP-1 inhibitor therapy. Breast Cancer Res Treat 2011; 127:861-869.

30. Ellsworth RE, Ellsworth DL, Love B, et al. Correlation of levels and patterns of genomic instability with histological grading of DCIS. Ann Surg Oncol 2007; 14: 3070-3077.

31. Heaphy CM, Bisoffi M, Joste NE, Genomic instability demonstrates similarity between DCIS and invasive carcinomas. Breast Cancer Res Treat 2009; 117: 17-24

32. Meeker AK, Hicks JL, Gabrielson E, et al. Telomere shortening occurs in subsets of normal breast epithelium as well as in situ and invasive carcinoma. Am J Pathol 2004; 164: 925-935.

33. van Hoesel AQ, Sato Y, Elashoff DA, et al. Assessment of DNA methylation status in early stages of breast cancer development. Br J Cancer 2013; 108: 2033-2038.

34. Deng CX, Scott F. Role of the tumor suppressor gene Brcal in genetic stability and mammary gland tumor formation. Oncogene 2000; 19: 1059-1064.

35. Schuetz CS, Bonin M, Clare SE, et al. Progression-specific genes identified by expression profiling of matched ductal carcinomas in situ and invasive breast tumors, combining laser capture microdissection and oligonucleotide microarray analysis. Cancer Res 2006; 66: 5278-5286.

36. Hernandez L, Wilkerson PM, Lambros MB, et al. Genomic and mutational profiling of ductal carcinomas in situ and matched adjacent invasive breast cancers reveals intra-tumour genetic heterogeneity and clonal selection. J Pathol 2012; 227: 42-52.

37. Heselmeyer-Haddad K, Berroa Garcia LY, Bradley A, et al Single-cell genetic analysis of ductal carcinoma in situ and invasive breast cancer reveals enormous tumor heterogeneity yet conserved genomic imbalances and gain of MYC during progression. Am J Pathol 2012; 181: 1807-1822.

38. Balleine RL, Webster LR, Davis S, et al. Molecular grading of ductal carcinoma in situ of the breast. Clin Cancer Res 2008; 14: 8244-8252.

39. Abdel-Fatah TM, Powe DG, Hodi Z, et al. Morphologic and molecular evolutionary pathways of low nuclear grade invasive breast cancers and their putative precursor lesions: further evidence to support the concept of low nuclear grade breast neoplasia family. Am J Surg Pathol 2008; 32: 513-523.

40. Masood S, Rosa M. Borderline breast lesions: diagnostic challenges and clinical implications. Adv Anat Pathol 2011; 18: 190-198.

41. Barnes NL, Ooi JL, Yarnold JR. Ductal carcinoma in situ of the breast. BMJ 2012; 344:e797.

42. Wapnir IL, Dignam JJ, Fisher B, et al. Long-term outcomes of invasive ipsilateral breast tumor recurrences after lumpectomy in NSABP B-17 and B-24 randomized clinical trials for DCIS. J Natl Cancer Inst 2011; 103: 478-488.
43. Emery LA, Tripathi A, King C, et al. Early dysregulation of cell adhesion and extracellular matrix pathways in breast cancer progression. Am J Pathol 2009; 175: 1292-1302.

\section{Address for correspondence}

Prof. Wenancjusz Domagala, M.D., Ph.D.

Department of Pathology

Pomeranian Medical University

Szczecin, Poland

Unii Lubelskiej 1

71-252 Szczecin, Poland

fax: +48914870032

e-mail: wenek@pum.edu.pl 